\title{
Erratum to: Equivalent alkane carbon number of crude oils: A predictive model based on machine learning
}

Benoit Creton ${ }^{1,3,}{ }^{*}$, Isabelle Lévêque ${ }^{1,3}$, and Fanny Oukhemanou ${ }^{2,3}$

${ }^{1}$ IFP Energies nouvelles, 1 et 4 avenue de Bois-Préau, 92852 Rueil-Malmaison, France

${ }^{2}$ Solvay-Laboratory of the Future, 178 avenue du Dr. Schweitzer, 33600 Pessac, France

${ }^{3}$ The EOR Alliance, www.eor-alliance.com

Accepted: 1 October 2019

Oil \& Gas Science and Technology - Rev. IFP Energies nouvelles 74, 30 (2019)

An error occurred in the version of the article OGST180315 available online, equation (5) should read:

$$
\begin{gathered}
\lambda_{0}=\text { Intercept }=-44.28, \\
\lambda_{1} \mathrm{G}_{1}=-0.39 \frac{\text { Aro. }}{\text { Asp. }} \\
\lambda_{2} \mathrm{G}_{2}=0.30 \exp \left(\frac{\text { Sat. }}{\text { API }- \text { Aro. }}\right), \\
\lambda_{3} \mathrm{G}_{3}=4.67 \times 10^{-5}\left(\text { API }^{3}+\text { Sat. }^{3}+\text { Res. }^{3}\right), \\
\lambda_{4} \mathrm{G}_{4}=55.12 \exp (-\exp (- \text { Aro. })),
\end{gathered}
$$

$$
\begin{gathered}
\text { instead of: } \\
\lambda_{0}=\text { Intercept }=-44.28, \\
\lambda_{1} \mathrm{G}_{1}=-0.39 \frac{\text { Aro. }}{\text { Asp. }} \\
\lambda_{2} \mathrm{G}_{2}=0.30 \exp \left(\frac{\text { Sat. }}{\text { API }- \text { Aro. }}\right), \\
\lambda_{3} \mathrm{G}_{3}=4.67 \times 10^{-5}\left(\mathrm{API}^{3}+\text { Sat. }^{3}+\text { Res. }^{3}\right), \\
\lambda_{4} \mathrm{G}_{4}=55.12 \exp (-\exp (\text { Aro. })) .
\end{gathered}
$$

\footnotetext{
* Corresponding author: benoit.creton@ifpen.fr
} 